\section{Judgments of slope variability in visual patterns*}

\author{
NANCY S. ANDERSON and AZRIEL ROSENFELD \\ University of Maryland, College Park, Md. 20742
}

Ss judged the similarity of pairs of patterns made up of $10 \times 10$ arrays of short line segments. Patterns differed with respect to the variability of occurrence of each of eight possible angles of orientation of the individual segments. The amount of information per line segment was the best linear predictor of the average similarity ratings. Other measures of statistical properties of these patterns are also described.

There are a multitude of studies in the literature that investigate how Ss make judgments about a stimulus that varies along some physical continuum. For example, Ss can be asked to judge the heaviness of a weight, the loudness of a tone, the brightness of a spot of light, the area of a circle, and so on. The stimuli used in such classical psychophysical studies are generally quite simple, and the magnitude to be judged is a physical quantity which varies along a single dimension.

A related area of investigation is that of how Ss make overall judgments about complex stimuli, where a stimulus consists of many parts, each of which varies along some common continuum. In particular, one can study how Ss estimate statistical properties of such stimuli, For example, suppose that a stimulus is a large array of small circles, each having a different luminance (or a different size, etc.). Here the Ss could be asked to estimate the "average" luminance (or size), or the tariability of the luminance (or size). Of particular interest in this connection are such questions as :

(1) How do Ss' judgments of "average," of variability, etc., relate to statistical measures such as the mean or median, the variance or standard deviation, and so on?

(2) Are these relationships similar for different physical variables (e.g., is judged variability of size related to size variance in the same way that judged variability of luminance is related to luminance variance), or are they different for different variables?

A number of earlier studies have dealt with overall judgments of complex stimuli. It has been shown [e.g., Attneave (1957); see Zusne $(1971$, p. 280$)$ for a summary of his

* This research was supported in part by NASA Grant NsG 398 and in part by NSF Grant GJ-32258X. The authors wish to thank Myrna Fivel and Anita Cunitz for their assistance in data collection, and John Pfaltz for the program for pattern generation. work and others'] that Ss' judgments of the similarity of complex forms are related to such properties as the number of angles in a form's contour and the variability of the magnitudes of these angles. Pollack (1971), Julesz (1962), and Pickett (1967) have studied judgments made on statistically generated dot patterns; for example, they have shown that the judged "coarseness" or "evenness" of such a pattern is related to the conditional probabilities with which dots and spaces follow dots and spaces. Stoloff (1969) studied judged variability using arrays of clusters of dots and arrays of small, simple shapes. He found that variability judgments were monotonically related to variance, but that a given difference in variance gave rise to a greater judged difference in variability for pairs of high-variance arrays than for pairs of low-variance arrays.

The present paper describes a study of variability judgments, using arrays of short line segments; the physical property which varied was segment slope. (The use of slope as a property introduces certain special problems about how to measure variability on a cyclic scale; these problems are described in the discussion section.)

\section{STIMULI}

The stimuli used in the present studies were $10 \times 10$ matrices of line segments in which each segment had one of eight possible orientations, from 22.5 to $157.5 \mathrm{deg}$ in equal steps. One of the four angles of $22.5,45$, 67.5 , and $90 \mathrm{deg}$ was specified as the "dominant" angle. For each choice of dominant angle, a series of seven patterns was generated; these patterns varied in terms of the probabilities of the remaining angles. For the most structured pattern (No. 1), the "dominant" angle occurred very often ( $7 / 8$ of the time) and each of the other angles occurred $1 / 56$ of the time. In the next most structured pattern (No. 2), the dominant angle occurred $6 / 8$ of the time and each of the other angles occurred $2 / 56$ of the time. The occurrence of the dominant angle and change in probability of the dominant angle occurred in steps of $1 / 8$, while that of the other angles occurred in steps of $1 / 56$. In the most random pattern (No. 7), each angle occurred equally often, $1 / 8$ of the time. Examples of the patterns used are shown in Fig. 1.

\section{PROCEDURE}

The patterns were constructed by a Calcomp plotter, which drew each $10 \times 10$ matrix of lines in a $9 \times 9 \mathrm{~cm}$ square, which was then mounted on a standard sheet of plain paper $(21.2 \times 27.8 \mathrm{~cm})$. The $\mathrm{E}$ sat opposite the $S$ and placed pairs of patterns in front of $\mathrm{S}$ for $.25 \mathrm{sec}(250 \mathrm{msec})$. All Ss had normal vision and viewed the pairs of patterns placed adjacent to one another from a viewing distance of $50 \mathrm{~cm}$ ( 20 in.).

Ss were shown samples of the patterns and told how the "structured" and "random" patterns were constructed. Ss were then shown pairs of patterns consisting of the most structured or most random as a standard paired with each of the other seven patterns in a series for each of the four dominant angles. Fifteen Ss
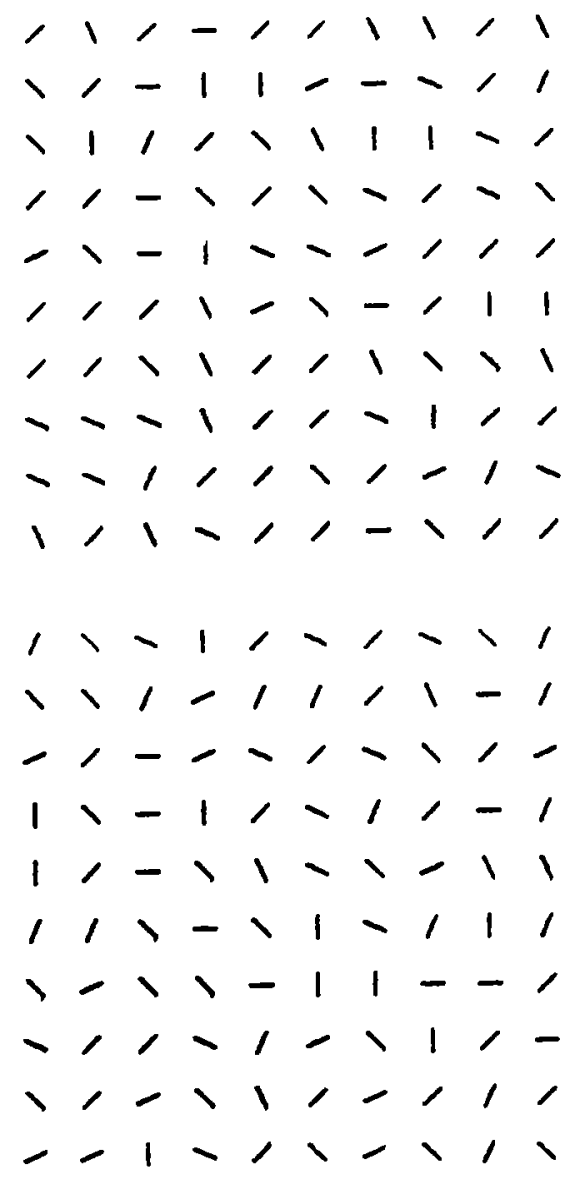

Fig. 1. Examples of patterns: (1) $45 \mathrm{deg}$ random compared to (2) Pattern 5. 

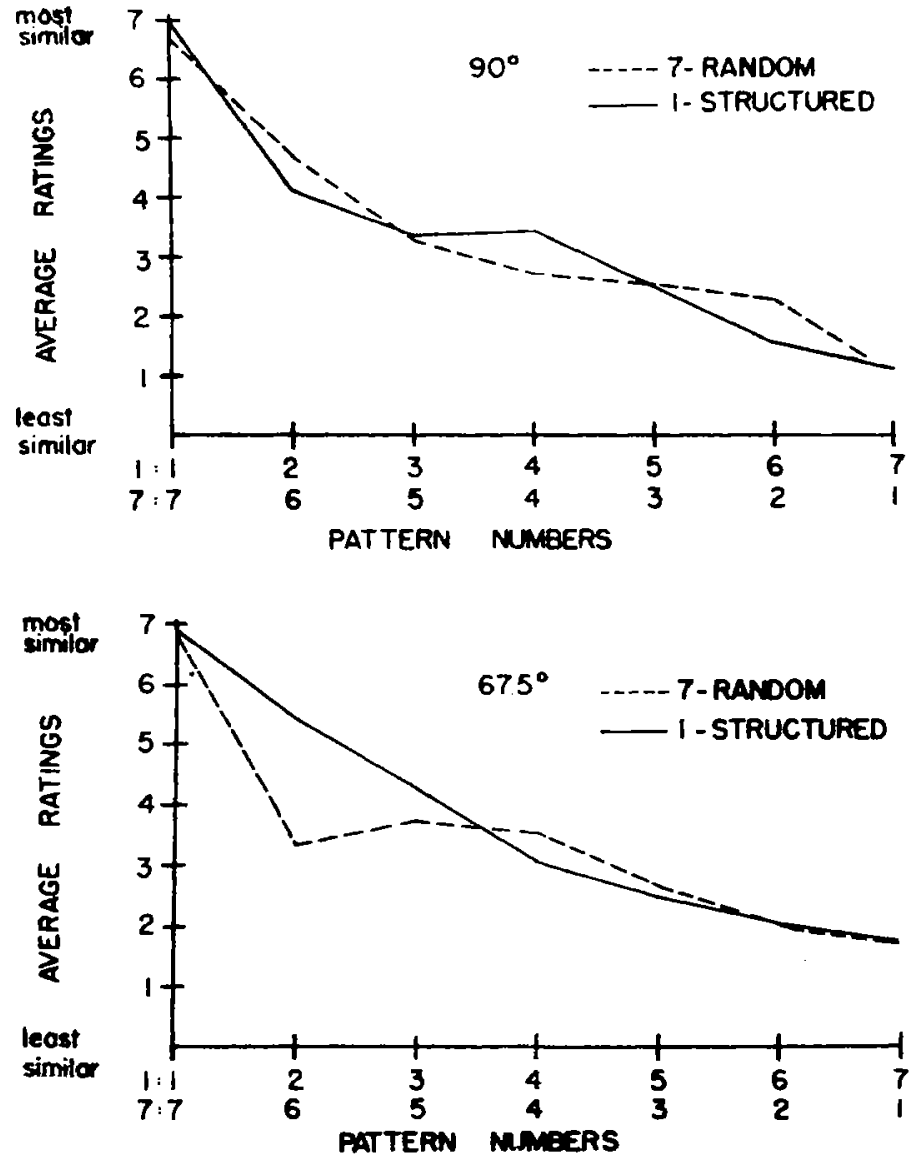

Fig. 2a. Average ratings of similarity by pattern number.

were asked to judge for each pair the similarity of the patterns with respect to variability of slopes on a $1-7$ scale, where $7=$ most similar and $1=$ least similar. Each pair of patterns for the series was presented to each $\mathbf{S}$ twice throughout a session. The average similarity of each pair for each $S$ was calculated and averaged for all 15 Ss.

\section{RESULTS}

Judged similarities of Patterns 2, .., 7 to the structured pattern (No. 1) and judged similarities of Patterns 1, ..., 6 to the random pattern (No. 7 ), averaged for $15 \mathrm{Ss}$, are plotted in Figs. 2a and $2 b$ for each of the dominant angles.

An interesting feature of Fig. 2 is that, except for the $90-\mathrm{deg}$ dominant angle, there appears to be a tendency for Ss to judge that the third most random pattern is more similar to the random pattern than is the second most random pattern (i.e., they judge that Pattern 5 is more similar to Pattern 7 than is Pattern 6). This may be explainable on the grounds that the visual system is less sensitive to oblique angles than to verticals (or horizontals) (Bouma \& Andriessen, 1968 ), so that the slight preponderance of the dominant angle in Patterns 6 and 5 is not noticed when the dominant is oblique and the similarity judgments are based on accidental local resemblances.

Statistical analyses of the average similarity ratings for adjacent pairs of patterns showed that the judgments of Pattern 5 as more similar to Pattern 7 than Pattern 6 were not significantly different, except when the dominant angle was $45 \mathrm{deg}$, which is an angle for which the visual system is least sensitive $(t=2.80, \mathrm{df}=14, \mathrm{p}<.05)$.

$$
\text { DISCUSSION }
$$

It would appear from the results of these experiments that $S s$ are indeed responding to the variability of the line segment slopes in the stimuli. It is more difficult, however, to specify how this variability might be measured.

One possible measure of variability is the average amount of information per line segment. It is well known that this is greatest when all angles are equally likely, i.e., when the variability is greatest, and that it decreases as one angle becomes dominant, i.e., as the variability decreases. The quantitative relation between information and variability has been described in detail bv Garner \& McGill (1956).

Let $p$ be the probability of an angle other than the dominant angle. There are seven such angles, and each conveys - $\log p$ bits of information, while the dominant angle occurs with probability $1-7 \mathrm{p}$ and conveys $-\mathrm{log}$ $(1-7 p)$ bits. Hence the average number of bits of information per line segment is given by $-7 p \log p-$ $(1-7 p) \log (1-7 p)$.

The correlations between average information per line segment and average similarity rating for each of the four dominant angles from 90 to $22.5 \mathrm{deg}$ were $.97, .90, .94, .93$ for Pattern 7 as standard and $.97, .99, .98$, .99 for Pattern 1 as standard. These correlations all exceed .9. (The absolute values of the correlation coefficients are given in all cases.) The fact that the correlations were lower (except for $90 \mathrm{deg}$ ) for the "random" vs the "structured" as a standard may be accounted for by the same explanation given previously that the visual system is less sensitive to oblique than to vertical angles.

Another possible approach to defining a measure of variability is to use the variance or standard deviation of the angle; this type of measure was used by Stoloff (1969). However, it is not clear how to define such measures for the present patterns, since we are dealing with a cyclic scale: $0 \mathrm{deg}$ is as close to $157.5 \mathrm{deg}$ as it is to $22.5 \mathrm{deg}$, and there is no meaningful "mean" angle. 1 Conceivably, when there is a dominant angle, one might measure variability about that angle, whereas when there is no dominant angle, variability about the vertical might be measured, since it is reasonable to assume (see Results section above) that vertical is a "preferred" orientation. In these terms, if we let $\alpha$ $=22.5 \mathrm{deg}$ and $p=$ the probability of an angle other than the dominant angle, the variance becomes

$$
2 \alpha^{2} p^{\prime}+2(2 \alpha)^{2} p+2(3 \alpha)^{2} p^{\prime}+(4 \alpha)^{2} p
$$

since there are two angles that differ from the dominant one (or from the vertical, in the completely random case of Pattern 7), by $\alpha$, two that differ by $2 \alpha$, two that differ by $3 \alpha$, and one that differs by $4 \alpha$. Note that this expression, which evaluates to $44 \alpha^{2} \mathrm{p}$, depends not only on $\mathrm{p}$, but also on the difference $(\alpha)$ between angles. The correlations between average similarity rating and variance, as well as between similarity and standard deviation (defined as the square root of variance), for each of the four dominant angles for Pattern 7 as a standard were $.94, .87, .93, .87$ (variance) and $.91, .84, .91, .83$ (standard deviation); for Pattern 1 as a 
standard they were $.94, .97, .97, .97$ (variance) and .97, .99, .98, .99 (standard deviation). These correlations are also high, but not as high as those for information per line segment. 2

Still another possibility is that the Ss may not be judging variability of slope at all, but rather may be judging the average number of nonparallel neighboring pairs of segments; this average is closely related to slope variability. Indeed, if a segment is at a nondominant angle, the probability of its having a particular neighboring segment parallel to it is $p$; if at the dominant angle, it is $1-7 \mathrm{p}$. The expected proportion of nonparallel pairs of segments is thus $1-\left[7 \mathrm{p}^{2}+\right.$ $\left.(1-7 p)^{2}\right]$.' The correlations between this quantity and average rating were also high, but again not as high as for the information measure. For Pattern 7 as a standard, the values were $.83, .77, .86, .74$, and for Pattern 1 as a standard, they were .96 , $.99, .98, .98$.

\section{CONCLUSION}

The data demonstrate that Ss can judge similarity of displays of arrays of line segments which differ according to the variability of the angles of the line segments. The ratings for different dominant angles in the arrays support previous work showing that the similarity judgments reflect less similarity when a vertical angle is dominant. Of the particular measures of the variability of the patterns, the average amount of information per line segment in the array was the best linear predictor of Ss' average similarity rating. Further research is needed to determine more precisely the appropriate measure of variability, since for the range of array differences used here, other measures of variability are also linearly related to the average amount of information per line segment.

\section{REFERENCES}

AtTreave, F. Physical determinants of the judged complexity of shapes. Journal
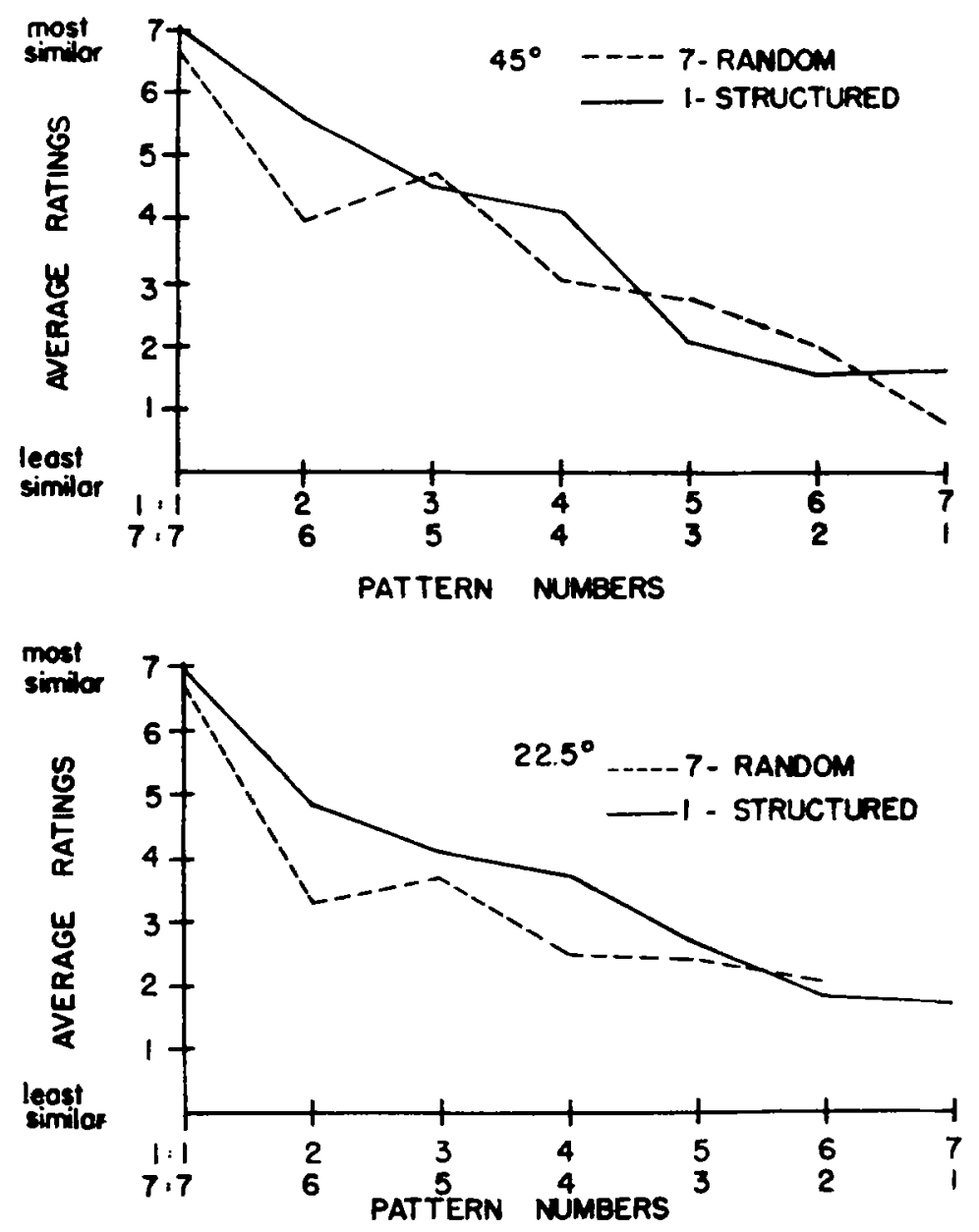

Fig 2b. Average ratings of similarity by pattern number.

of Experimental Psychology, 1957, 53, 221-227.

BOUMA, H. \& ANDRIESSEN, J. J Perceived orientation of isolated line segments. Vision Research, 1968, 8 , 493-507.

GAR NER, W. R., \& McGILL, W. J. Relation between uncertainty, variance, and correlation analyses. Psychometrika, $1956,21,219-228$.

JULESZ, B. Visual pattern discrimination. IRE Transactions on Information Theory, 1962, IT-8, 84-92.

PICKETT, R. M. The perception of random visual texture. In W. Wathen-Dunn (Ed.). Models for the perception of speech and visual form. Cambridge, Mass: M.I.T. Press, 1967.
P OLLACK, I . Perception of two-dimensional Markov constraints within visual displays. Perception \& Psychophysics, 1971, 9, 461-464.

STOLOFF, P. H. Detection and scaling of statistical differences between visual textures. Perception \& Psychophysics, $1969,6,333-336$.

ZUSNE, L. Visual perception of form. New York: Academic Press, 1970.

\section{NOTES}

1. An analogous situation would arise if we were scaling variability of color.

2. Since the sample size used to calculate the correlations was so small, it seemed unadvisable to test for significance of differences between correlations. 